\title{
"In un placete de La Mancha of which nombre no quiero remembrearme": Don Quijote en spanglish y los desafíos de la traducción bilingüe
}

\author{
Marco Kunz \\ Otto-Friedrich-Universität Bamberg
}

Spanglish, the mixing of Spanish and English in oral and written communication, has an increasing importance as a literary code. Challenged by derogatory comments on the poor aesthetic possibilities of Spanglish, Mexican American scholar Ilan Stavans translated the first chapter of Cervantes' Don Quijote into a mixed language made of English, Spanish and hybrid words. The result provoked the hilarity of many readers, but also the indignation of Castilian purists who opposed the invasion of their mother tongue by a foreign language and the desecration of the most outstanding monument of the Hispanic Culture, while American Chicanos criticized the quality of the translation, arguing that Stavans' Spanglish fails to reflect accurately the reality of bilingual speech in daily life. In my paper I propose some reflections about this controversy and I try to analyse Stavans' intentions and strategies in this translation of a canonical classical text into a non-normative and highly stigmatized variety of spoken language. As it is directed to a bilingual audience, this translation loses its principal raison d'être, that is to make the text understandable for foreign readers, but it fulfils other functions.

\section{El spanglish, ¿problema o problemática?}

La mezcla de dos idiomas, fenómeno frecuente en el habla de individuos bilingües, siempre ha sido objeto de la censura por parte de personas que la ven como degeneración, bastardización, babelización o invasión de la lengua materna por un agresor extranjero más poderoso. Mientras que en la época de Antonio de Nebrija (1492) el español era la lengua 'compañera' del imperio en el que nunca se ponía el sol, el nuevo Empire de hoy se expresa en inglés, de modo que los no anglófonos creen a menudo encontrarse en una situación amenazada y adoptan actitudes de autodefensa. El spanglish, producto del mestizaje de estas dos lenguas imperiales, la antigua y la actual, es una forma híbrida hablada y, a veces, escrita por los latinos en Estados Unidos, un español primordialmente oral, lleno de anglicismos y neologismos, que se observa cada vez más en los medios de comunicación impresos (publicidad, periódicos) y audiovisuales (canciones pop, hip hop, rap, emisiones de televisión, programas de la radio, etc.) que se dirigen a un público bilingüe en una variedad lingüística que éste usa en su vida cotidiana con la mayor naturalidad, pero que está proscrita por la norma lingüística. 
Si hablamos de spanglish o espanglish (con la $e$ protética del español), espanglis (imitación de cómo se pronunciaría con acento español) o espanglés ${ }^{1}$ (forma híbrida, análoga a expresiones semejantes como franglais o portuñol ${ }^{2}$ ), en todos estos casos las palabras llevan en sí la connotación negativa que tradicionalmente estigmatiza cualquier tipo de mestizaje. En las últimas décadas, bajo el efecto de la mundialización, las reivindicaciones de las minorías culturales y el relativismo del pensamiento posmoderno y postestructuralista, las nociones de mestizaje e hibridismo han empezado a adquirir sentidos positivos, y la sociolingüística moderna ya no estudia fenómenos como la alternancia códica (code-switching) o el préstamo desde una perspectiva normativa y purista, sino que los considera como manifestaciones de una competencia comunicativa enriquecedora que los hablantes monolingües no poseen. Sin embargo, esta tendencia a la revaloración de la mezcla choca contra prejuicios, mala información, rechazo político, aprensiones xenófobas y muchos más obstáculos. Hay un enfrentamiento de intereses en cuyo contexto el hibridismo de las dos lenguas resulta reprobable porque se teme que la indulgencia ante la infracción debilite la propia posición. El avance del español, la lengua minoritaria más importante en Estados Unidos (ya son aproximadamente 40 millones los hispanohablantes en el país), preocupa a algunos americanos anglófonos, como el politólogo Samuel Huntington que recientemente provocó una gran polémica con su ensayo "El reto hispano", ${ }^{3}$ donde expresa sus temores ante la inmigración latinoamericana que, según él, amenaza la esencia de la tradición estadounidense (i.e. protestante, individualista, anglófona), y repercute en medidas represivas como la política del English Only o English First. Por otra parte, hay latinos en Estados Unidos que, ansiosos de defender su hispanidad (o, en un sentido más estricto, su mexicanidad, cubanidad, etc.) contra la fuerza de absorción de la cultura norteamericana, rechazan el spanglish porque representa para ellos un síntoma alarmante de la progresiva pérdida de identidad cultural y la ocupación creciente de su territorio lingüístico por el inglés. Otros argumentan, y no sin cierta razón, que el spanglish es la consecuencia del bajo status socio-económico de los hispanohablantes en Estados Unidos, un síntoma de su escolarización insuficiente, su incultura debida a la pobreza y la discriminación, y que sólo el aprendizaje del inglés correcto y, en segundo lugar, del castellano normativo les permitirá salir del gueto. Visto así, el spanglish literario, en el mejor de los casos, sirve de divertimiento para los happy few perfectamente bilingües, mientras que, para la mayoría de los que lo emplean en su vida diaria, el spanglish hablado significa un síntoma y, al mismo tiempo, una causa de su exclusión social.

\section{Ilan Stavans, "celui par qui le scandale arrive"}

Pocos se muestran tan entusiastas del spanglish como su defensor más conocido, Ilan Stavans, un mexicano judío con antepasados rusos y polacos (su 
verdadero apellido es Stavchansky), nacido en 1961 en el Distrito Federal y emigrado, a la edad de veinticuatro años, a Estados Unidos, donde trabaja actualmente en Amherst College como profesor de cultura latinoamericana (cf. la introducción a Stavans 2003a y el ensayo autobiográfico Stavans 2003b). Stavans no vacila en hablar de The Making of a New American Language (Stavans 2003a) y fomenta el spanglish con la publicación de antologías (Stavans 2003c), ensayos y un diccionario que recoge miles de palabras. "Prestarle [al spanglish] la atención que se merece, en lugar de verlo con displicencia y animosidad", es la intención de Stavans. El spanglish lo fascina por su "gran creatividad" y su "enorme espíritu de improvisación", y reivindica el derecho al mestizaje: "así como el mestizo es impuro, el spanglish es impuro y no debe avergonzarse por ello" (cit. por Marín 2002). Stavans define el spanglish como "[t]he verbal encounter between Anglo and Hispano civilizations" (Stavans 2003a: 5), y lo hace después de haber ponderado los argumentos en pro y en contra de otra formulación, que habría dicho clash en vez de encounter y languages por civilizations. Aunque sabe bien que el spanglish constituye para muchos inmigrantes y sus hijos una trampa, un obstáculo en el camino hacia la integración en la sociedad norteamericana, proceso que pasa necesariamente por la adquisición exitosa del inglés - "English is the door to the American Dream. Not until one masters el inglés are the fruits of that dream attainable" (Stavans 2003a: 3) - , Stavans aprendió a apreciar e incluso admirar el spanglish, llegando a compararlo con el jazz, esa música que nació de la incultura musical y conquistó el mundo con sus nuevos ritmos y sonoridades.

De todos sus textos escritos en spanglish, el que más polémicas ha suscitado es sin duda la traducción del comienzo del Quijote que hizo Stavans aceptando el desafío lanzado por un español que, en una discusión en la radio catalana, en verano de 2002, había dicho despectivamente que no se podía tomar en serio el spanglish hasta que produjera una novela como el Quijote. Stavans pasó al contraataque y escandalizó a sus detractores con una casi sacrílega versión bastarda de la obra maestra de Cervantes: primero, improvisó algunas frases y después, cediendo a la demanda, tradujo un capítulo entero que se publicó, parcial o íntegramente, en varios periódicos (primero el 3 de julio de 2002 en La Vanguardia de Barcelona) y provocó una “controversia internacional", según los términos del propio Stavans (2003a: 252). Dado que el spanglish sirve, ante todo, como vehículo oral de la comunicación hablada por individuos de distintos orígenes nacionales, Stavans optó por un spanglish mixto, panamericano, comparable a la mezcla de variedades que se oye cada vez más en los programas televisivos y radiofónicos en Estados Unidos, un spanglish no estardandizado, permeado por todas las modalidades que conocía, con el propósito de crear un texto legible tanto para hablantes de una variedad del espanglés como para anglófonos e hispánicos más o menos bilingües. Y no le importa que sea un spanglish artificial: "Until and unless Spanglish moves from the oral to the written mode" - and it's showing signs of doing so already - any literary attempt is, inevitably, una afectación" (Stavans 2003a: 252). 
Mientras que a Stavans su traducción le pareció un experimento que califica de joyful y joyceano, las reacciones vehementes de muchos lectores muestran que no todos supieron apreciar ni la intención ni el resultado y que tomaron muy en serio algo que, a lo mejor, no es más que un juego lingüístico y literario divertido. “¡Horror!, el Quijote en spanglish” (Ferrer Rodríguez 2002), tituló La Jornada de México. Otro comentarista escribió indignado en Libertad Digital: "No contento con abanderar la destrucción del inglés y del español en que consiste esta jerigonza denominada spanglish, un tal Ilan Stavans acaba de transcribir El Quijote en este mejunje lingüístico" (Ruiz Portella 2002). El sacrilegio de Stavans consiste no sólo en defender la bastardización de las lenguas y postular la legitimidad de la hija natural, repudiada por los puristas, sino también en no respetar la jerarquía y pretender darle al spanglish, una variedad considerada inferior, la dignidad de un idioma literario, como le reprochó un crítico:

a lo que sobre todo no hay derecho es a considerar que quienes practican tal jerga están hablando (al cabo de tan sólo diez o veinte años de mescolanza) una nueva lengua; una lengua a la que hasta merece que se traduzca una de las más grandes obras de todos los tiempos. (Ruiz Portella 2002)

"No hay derecho": la frase es reveladora, pues el spanglish está en el centro de un debate sobre la propiedad cultural. Tal como lo concibe Stavans, se legitima, precisamente, por el derecho - vivido, hablado y escrito por más de 40 millones de hispanohablantes en Estados Unidos - de usar libremente la lengua propia, o mejor dicho, las lenguas propias: "Estamos ante una generación que no pide disculpas for the language they are using" (Marín 2002), contestó Stavans a Roberto González Echeverría, quien considera el spanglish como una invasión del español por el inglés que no respeta su esencia y dignidad. Ante la legislación lingüística, Stavans defiende la lengua como "the most open and democratic manifestation of spirit" (Marx \& Escobar Ulloa 2004). Su traducción del Quijote tiene antecedentes importantes en su enseñanza universitaria: como cuenta en la introducción a su diccionario del spanglish (Stavans 2003a: 15-17), sus estudiantes empezaron a traducir a la "jerga loca" textos de alto prestigio en la sociedad anglófona estadounidense, como la Declaración de independencia o la Constitución de los Estados Unidos, para después aplicar el mismo método de hibridación a incipits de famosas novelas. Stavans interpreta esta traducción lúdica como la reapropiación de los artefactos culturales mayores que afectan a la vida de los Hispanics, como una estrategia de desalienación: espanglizando los textos norteamericanos, los latinos estadounidenses superan su complejo de inferioridad, rompen el tabú del mestizaje, le quitan sus estigmas vergonzosos y logran asumirlo como su esencia positiva.

También algunos críticos menos puristas le reprochan a Stavans el intento de traducir el Quijote: así David Roas (2005: 10), quien calificó la empresa de inútil e innecesaria y el producto de "frankensteiniano", un monstruo compuesto de miembros de cuerpos diferentes. Como la traduc- 
ción sirve para favorecer la comunicación intercultural y permitir la lectura de obras literarias cuya lengua original no se comprende, Roas se pregunta para qué se traduce un texto que el público meta, necesariamente bilingüe, puede leer en una de las dos lenguas, y considera la iniciativa de Stavans como un juego de intelectuales cuya posición privilegiada poco tiene en común con la realidad diaria de la mayoría de los hispanohablantes estadounidenses, generalmente de baja extracción social y con deficiencias culturales evidentes. Los autores en spanglish, en cambio, opina Roas (2005: 10), son "unos pocos tipos cultos que se dirigen a otros pocos tipos cultos como ellos que seguramente ya han leído el Quijote en español o al menos han podido hacerlo". Roas tiene razón con sus reparos, pero no parece haber entendido - o se niega a aceptarlo - que la intención de Stavans no consiste en absoluto en colmar una laguna y satisfacer una necesidad, sino, al contrario, en demostrar una posibilidad, reaccionando a una provocación que le negaba al spanglish literario su derecho a ser. Es una rebelión que no se dirige contra Cervantes ni la lengua española, sino contra un modelo inalcanzable y alegado como argumento para descalificar la literatura en spanglish: el Quijote, con toda la admiración que merece, ya no puede servir como ejemplo imitable de estilo y lengua, pues resultaría absurdo escribir hoy día con el léxico y la sintaxis de principios del siglo XVII (de ahí viene precisamente el carácter descabellado de la empresa de Pierre Menard en la celebérrima "ficción" de Jorge Luis Borges). Y, en efecto, sólo disfruta de la lectura del Quijote en spanglish quien lo compare con el original y sepa apreciar las distorsiones y metamorfosis que Stavans le ha hecho sufrir a las palabras del texto cervantino.

El objetivo de esta traducción 'inútil' no consiste en volver comprensible el original para nuevos lectores, sino en mostrar el potencial expresivo del code-switching y la hibridación de lenguas, en reivindicar una práctica comunicativa colectiva de millones de hablantes, jugar con las posibilidades de mezcla, hacer reír de la deformación excesiva que sufre el original y, last but not least, contribuir a una autoafirmación no acomplejada de los lectores bilingües. Si, para los estudiantes de Stavans, el hecho de traducir al spanglish comienzos de novelas anglosajonas famosas tuvo un efecto de desinhibición ante la presión del idioma dominante en Estados Unidos, el Quijote en spanglish apunta a liberarlos de la humillante censura de su 'mal español' por parte de hispanohablantes peninsulares o latinoamericanos, discriminación contra la que se revoltó ya Gloria Anzaldúa (1999: 76) en su célebre ensayo bilingüe Borderlands/La frontera: "nos quieren poner candados en la boca. They would hold us back with their bag of reglas de academia". La escritora chicana reivindicó el derecho a la expresión mixta, pues sólo si no tiene que optar constantemente por una u otra lengua para satisfacer las exigencias de los monolingües, el individuo bilingüe encuentra un modo adecuado de expresarse:

Until I am free to write bilingually and to switch codes without having always to translate, while I still have to speak English or Spanish when I would rather speak Spanglish, and as long as I have to accommodate the English speakers 
rather than having them accommodate me, my tongue will be illegitimate. (Anzaldúa 1999: 81)

\section{3. "Errores" creativos y literariedad}

Sin embargo, hay también espanglohablantes, como el periodista chicano Rigoberto Anzaldúa (2003: 40), que se sintieron provocados, insultados, humillados incluso por la "jerigonza" que, según él, presenta Stavans como el spanglish por antonomasia y por las "tonterías" que aquél afirma al respecto. Se acusa a Stavans de escribir sobre el spanglish desde "la protección de instituciones o condiciones privilegiadas", sin vivirlo "en las calles, diariamente". La crítica de Rigoberto Anzaldúa se dirige no tanto contra la mezcla de las dos lenguas, sino contra las insuficiencias de una traducción escrita en un spanglish lleno de "errores", que no tiene nada que ver con el discurso bilingüe real. Para demostrarlo, analiza la primera frase, que en el original cervantino reza así:

En un lugar de la Mancha, de cuyo nombre no quiero acordarme, no ha mucho tiempo que vivía un hidalgo de los de lanza en astillero, adarga antigua, rocín flaco y galgo corredor. (Cervantes 1998: vol. I, 35)

En la versión de Stavans, el incipit del Quijote suena así:

In un placete de La Mancha of which nombre no quiero remembrearme, vivía, not so long ago, uno de esos gentlemen who always tienen una lanza in the rack, una buckler antigua, a skinny caballo y un greyhound para el chase. (Stavans 2003a: 253)

Para Rigoberto Anzaldúa (2003: 40), esta frase inicial de la traducción basta para demostrar el fracaso de la empresa de Stavans, ya que "presenta al 'espanglohablante' como un imbécil total, incapaz de recurrir a una base lingüística compartida". Anzaldúa peca aquí de la típica ceguera del que no se da cuenta de que la capacidad de recurrir no a una, sino a tres bases lingüísticas compartidas (el español, el inglés y el spanglish) no es precisamente un síntoma de pobreza expresiva, sino todo lo contrario: Shana Poplack demostró que la alternancia códica en el interior de la frase exige una gran habilidad bilingüe ya que presupone un buen dominio de las reglas sintácticas de ambas lenguas: "Code-switching, [...] rather than representing deviant behaviour, is actually a suggestive indicator of degree of bilingual competence" (Poplack 1980: 616). Sin embargo, la crítica que hace Anzaldúa del spanglish de Stavans merece ser mirada más de cerca:

El más inepto "espanglohablante" recurriría a "lugar" en vez de "un placete", a "cuyo nombre" en vez de "of which nombre"; y remplazaría "not so long ago" con "hace poco" (nunca el "ha mucho tiempo" original). Ahora, el "remembrearme" se aproxima a una posible expresión "bilingüe", aunque la 
práctica prueba que "remembrarme" sería una transcripción más exacta. Tal vez lo más revelador de la falta de consistencia traductora de Stavans es el uso del pronombre relativo who después de "uno de esos gentlemen". El uso es gramaticalmente correcto, en inglés, pero un "espanglohablante" emplearía la fórmula that. Y si el traductor ya había podido decir "vivía", ¿por qué acude más adelante a "livin' with él..."? Si es verdad que la práctica del spanglish frecuentemente significa el cambio de registro casi de oración a oración, cualquier conocedor experimentado sabe también que los extremos que muestra esta traducción son falsos, inexactos, exagerados, y, francamente, estúpidos. (Anzaldúa 2003: 40)

Un auténtico "espanglohablante", opina Anzaldúa, nunca hablaría así, y enumera varios ejemplos - errores, diría él - de la expresión poco natural que afea y desautoriza la traducción de Stavans. Ahora bien, como señala Ernst Rudin en su estudio del español en la novela chicana, la mímesis lingüística no es y no puede ni quiere ser una copia lingüísticamente fiel de enunciados espontáneos reales, y los procedimientos que crean este lenguaje obedecen más a convenciones literarias que a las leyes que rigen la lengua hablada (Rudin 1996: 15-24). El principal argumento que blande Rigoberto Anzaldúa contra la traducción de Stavans es que nadie diría esto, pero la realidad lingüística desmiente el afán normativo del periodista chicano: placete, por ejemplo, sí se emplea, y Stavans (2003a: 193) lo registra en su diccionario ("placete (pla-ZE-te), n., m., place, site. 'Nos conocimos en un placete de San Antonio"'), lo que significa que encontró un mínimo de tres ejemplos en el uso escrito u oral. Que remembrar se usa con más frecuencia que remembrear lo sabe también Stavans, pues la primera variante es la que incluyó en el diccionario: la crítica de Anzaldúa revela, pues, su conocimiento insuficiente de los estudios de Stavans sobre el spanglish. Si el traductor emplea tanto vivía como livin', no lo hace por inconsecuencia o incapacidad de decidirse por una de las dos lenguas, sino justamente porque el bilingüismo no lo obliga a tomar tal decisión y le ofrece, en cambio, una mayor variedad sinonímica, y quizás también porque uno de los mayores exitazos recientes del spanglish pop fue "Livin" la vida loca" de Ricky Martin. Sin duda, los cambios de código abundan demasiado en el texto de Stavans y se producen en posiciones muy poco probables en una conversación bilingüe real (p. ej. preposiciones o determinantes sueltos, cf. Poplack 1980: 602) y a menudo no parecen muy motivados o incluso innecesarios, pues no se explican por ninguna de las causas principales del code-switching, v. gr. la connotación cultural, la muletilla, el comentario, la cita, la repetición traducida, la puesta de relieve, el triggering (i.e. un elemento ambiguo, perteneciente a los dos sistemas lingüísticos, provoca un cambio de lengua), etc. (cf. Pfaff 1979, Poplack 1980, Gumperz 1982, Clyne 1987). ¿Por qué, p. ej., se sustituye flaco, palabra común y corriente, por skinny?: pues, simplemente porque es posible, y porque a Stavans le interesa aumentar la diferencia entre el texto original cervantino y su versión en spanglish, es decir, no sólo traduce lo que hay que traducir (sabe muy bien que, en rigor, la traducción es superflua), sino todo lo que cree que se puede. 
¿Cómo procedió Stavans al traducir el Quijote? Al comparar el original con la versión en spanglish, constatamos que, movido por el afán de maximizar la diferencia, Stavans sustituyó más de dos tercios de las palabras de Cervantes por expresiones inglesas (p. ej. gentlemen por hidalgo, rack por astillero, buckler por adarga, greyhound por galgo), híbridas (placete 'lugar' del inglés place más sufijo español -ete, remembrear 'acordar' derivado del inglés remember más el sufijo -ear) o sinónimos castellanos (caballo por rocín). Para diferenciar los distintos tipos de alteraciones del texto original, marco las palabras mantenidas del original mediante negritas, las expresiones inglesas en cursiva, los spanglishismos (i.e. formas híbridas) en cursiva subrayada, y subrayo las equivalencias españolas que reemplazan términos de la misma lengua:

In un placete de La Mancha of which nombre no quiero remembrearme, vivía, not so long ago, uno de esos gentlemen who always tienen una lanza in the rack, una buckler antigua, a skinny caballo $\mathbf{y}$ un greyhound para el chase. (Stavans 2003a: 253)

Algunas de estas sustituciones son, sin duda, puramente caprichosas (p. ej. of which por de cuyo, skinny por flaco), pero otras tienen una motivación evidente: muchos términos del español del siglo XVII del texto original deben de resultar incomprensibles tanto a los hispanohablantes de la primera generación de inmigrantes, que a menudo llegaron a Estados Unidos con poca formación escolar y un bagaje cultural limitado, como a sus hijos, nietos, bisnietos, etc., que dominan suficientemente una variedad coloquial del español del siglo XX para el uso casero, pero que adquirieron sus conocimientos de la historia en la escuela anglófona y, por tanto, ignoran el español de siglos pasados, de modo que probablemente desconocen palabras como hidalgo, astillero, adarga, rocín y galgo, aunque no sus equivalentes ingleses modernos o, en el caso de rocín, sinónimos españoles más corrientes como caballo. Por supuesto, no es necesario traducir el Quijote a una variedad de la misma lengua en la que está escrito, pero el texto de Cervantes sí precisa de muchas explicaciones para que lo comprendan los hispanohablantes de hoy: no hay que ser latino en Estados Unidos para no entender el texto cervantino: ¿cuántos españoles saben todavía que una adarga es un "escudo de cuero, ovalado o de forma de corazón" (Real Academia Española 2001: vol. I, 42) y un astillero una "percha en que se ponen las astas o picas y lanzas" (Real Academia Española 2001: vol. I, 233)? Normalmente, las notas a pie de página y los glosarios de las ediciones críticas cumplen la función metalingüística de explicar tales palabras caídas en desuso: Stavans, en cambio, actualiza el vocabulario para los lectores modernos eligiendo las equivalencias en el acervo léxico de los dos idiomas que se funden en el spanglish.

Para hacer más visibles las diferencias entre el original y la traducción de Stavans cotejamos en las listas siguientes las palabras de la segunda y tercera frase del Quijote con las equivalencias en spanglish: 


\begin{tabular}{|c|c|}
\hline Cervantes & Stavans \\
\hline Una & $A$ \\
\hline olla & cazuela \\
\hline de algo & with \\
\hline más & más \\
\hline vaca & beef \\
\hline que & than \\
\hline carnero, & mutón, \\
\hline salpicón & carne choppeada \\
\hline $\begin{array}{l}\text { las más noches, } \\
\text { duelos y quebrantos }\end{array}$ & $\begin{array}{l}\text { para la dinner, } \\
\text { un omelet }\end{array}$ \\
\hline $\begin{array}{l}\text { los sábados, } \\
\text { lentejas }\end{array}$ & 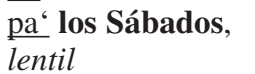 \\
\hline $\begin{array}{l}\text { los viernes, } \\
\text { algún }\end{array}$ & $\begin{array}{l}\text { pa' los Viernes, } \\
\text { y algún }\end{array}$ \\
\hline palomino & pigeon \\
\hline de & $\underline{\text { como }}$ \\
\hline añadidura & delicacy especial \\
\hline $\begin{array}{l}\text { los domingos } \\
\text { consumían }\end{array}$ & $\begin{array}{l}\text { pa ' los Domingos, } \\
\text { consumían }\end{array}$ \\
\hline las tres & $\{\varnothing\}$ tres \\
\hline partes & cuarers \\
\hline de su & de su \\
\hline hacienda. & income. \\
\hline El resto della & El resto $\{\varnothing\}$ \\
\hline concluían & 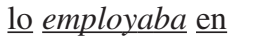 \\
\hline sayo & una coat \\
\hline de & de \\
\hline velarte & broadcloth \\
\hline calzas & y en soketes \\
\hline de & de \\
\hline velludo & velvetín \\
\hline para & $\underline{\mathrm{pa}^{6}}$ \\
\hline $\begin{array}{l}\text { las fiestas, } \\
\text { con }\end{array}$ & $\frac{\text { los holidays, }}{\text { with }}$ \\
\hline sus & sus \\
\hline pantuflos & slippers \\
\hline $\begin{array}{l}\text { de lo mesmo, } \\
\text { y }\end{array}$ & $\frac{\text { de combinar, }}{\text { while }}$ \\
\hline los días & los otros días \\
\hline de entresemana & de la semana \\
\hline se honraba con & él cut a figura de \\
\hline su vellorí de lo más fino. & de los más finos $c$ \\
\hline
\end{tabular}

Stavans tradujo las 66 palabras del texto cervantino mediante 75 palabras, de las cuales 24 se han conservado idénticas al original, 26 son unidades léxicas españolas diferentes, 19 inglesas y en seis casos se trata de formas híbridas (tres de ellas registradas en el diccionario de Stavans): mutón, choppeada, ${ }^{4}$ cuarers, employaba,${ }^{5}$ soketes,${ }^{6}$ velvetín, compuestas de una base lexemática inglesa más sufijos y rasgos fonéticos hispánicos. Se trata, por supuesto, de un fragmento muy breve, pero suficiente para ilustrar el trilin- 
güismo de la traducción (¿o habría que decir reescritura plurilingüe?) de Stavans.

Sin embargo, en algo tiene razón Rigoberto Anzaldúa: nadie habla este spanglish. Ahora bien, en el Siglo de Oro seguramente nadie hablaba como el Quijote de Cervantes, puesto que se trata de una obra literaria con un estilo muy elaborado y una lengua claramente diferenciada de la oralidad, pese a los numerosos diálogos que hay en esta novela (aunque no en el capítulo traducido por Stavans). Anzaldúa alega la oralidad real como instancia normativa para censurar un spanglish que no sólo no reconoce ninguna norma, sino que, al hacerse literario, se ha independizado de su origen oral. Parece que Anzaldúa no se percata de que el propósito estético de este spanglish específico no es mimético-realista, sino lúdico, y que no aspira a reproducir el habla de la calle, sino a desarrollar el potencial literario de un spanglish emancipado de las limitaciones de la oralidad y libre de combinar las lenguas. ¿No deberíamos considerar los “errores” que, según Anzaldúa, delatan la ineptitud del traductor, como señales de la literariedad del spanglish escrito por Stavans? Anzaldúa critica la versión de Stavans en el nombre de una supuesta norma del uso, una norma no codificada que él pretende conocer mejor que el mexicano Stavans, inmigrado como adulto hace una veintena de años, mientras que éste procura escribir en una hipotética nueva lengua literaria que (todavía) no posee un mínimo de codificación, de modo que el traductor tiene que inventársela, como también tienen que hacerlo los escritores norteamericanos que intentan crearse su propio spanglish en sus novelas, cuentos, poesías, ensayos, y que así contribuyen al Making of a New American Language.

\section{Conclusión}

Toda la polémica entorno al spanglish nos recuerda la crítica de la norma centralista castellana que formuló Juan Goytisolo en Reivindicación del Conde don Julián y Juan sin Tierra: los autodeclarados amos de la lengua y propietarios de sus "derechos reservados en todos los países", "estudiosos, licenciados, vates, sabios, expertos, peritos esgrimen sus títulos de dominio, posesión, usufructo" (Goytisolo 2001: 225), mientras que en la realidad lingüística cunde "el abla ef-fetiba de miyone de pal-lante que diariamente lamplean sin tenén cuenta er código pená impuet-to por su mandarinato" (Goytisolo 1977: 304). No debería olvidarse que el mismo Cervantes nos quería hacer creer que su Quijote no era más que la traducción de un original escrito por Cide Hamete Benengeli en árabe, la lengua del antagonista cultural principal en la época, del rival en la lucha por la hegemonía en el Mediterráneo y el opositor ideológico. El spanglish, objeto de un debate más emotivo que racional, se ofrecería como oferta reconciliatoria entre los nuevos imperios enfrentados, y la traducción del Quijote ironiza tanto acerca del purismo castellano, inapropiado a la realidad lingüística americana, como subvierte la doctrina del english only. Es un acto de libertad contra la impo- 
sición de normas monolingües a individuos cuya normalidad expresiva no se limita a una sola lengua ni tiene por qué separar estrictamente los dos idiomas, que en vez de la disyunción either or puede optar por la conjunción either and (Sommer \& Vega-Merino 1998). Y la libertad, le dijo Don Quijote a Sancho en el capítulo LVIII de la segunda parte, "es uno de los más preciosos dones que a los hombres dieron los cielos" (Cervantes 1998: 1094). O para decirlo en lunfardo, otra variante no normativa a la que es también totalmente inútil, pero sí placentero y refrescante, traducir la obra maestra de Cervantes: "la libertad es un batacazo bien debute que les enchufaron de garrón a los quías" (versión de Fernanda Nussbaum, en Alvar \& Talens 2004: 137).

\section{Bibliografía}

Alvar, Carlos \& Jenaro Talens (eds) (2004). Aquel a quien la fama quiere dalle el nombre que se tiene merescido. 105 versions et 16 illustrations d'un fragment de Miguel de Cervantes. Genève: El Dragón de Gales.

Anzaldúa, Gloria (1999). Borderlands / La Frontera. The New Mestiza. San Francisco: Aunt Luke Books.

Anzaldúa, Rigoberto (2003). "Los artistas del hambre". Lateral 10(97), 40.

Cervantes, Miguel de (1998). Don Quijote de la Mancha. 2 vols. Barcelona: Instituto Cervantes \& Crítica.

Clyne, Michael (1987). "Constraints on Code-switching: How Universal Are They?". Linguistics 25, 734-769.

Étiemble, René (1964). Parlez-vous franglais? Paris: Gallimard.

Fairclough, Marta (2003). "El (denominado) Spanglish en Estados Unidos: polémicas y realidades". Revista Internacional de Lingüística Iberoamericana 1(2), 185-204.

Ferrer Rodríguez, Eulalio (2002). “¡Horror!, el Quijote en spanglish”. La Jornada, 20.09.2002. On line en: http://www.jornada.unam.mx/2002/sep02/020920/006a1pol.php?origen=index.html (consultado 15.02.2005).

Goytisolo, Juan (1977). Juan sin Tierra. Barcelona: Seix Barral.

Goytisolo, Juan (2001). Don Julián. Barcelona: Galaxia Gutenberg / Círculo de lectores.

Gumperz, John J. (1982). Discourse Strategies. Cambridge: Cambridge UP.

Huntington, Samuel P. (2004). Who Are We? America's Great Debate. London: Simon \& Schuster.

Marín, Pablo (2002). "Una quijotada”. Qué pasa, 26.07.2002. On line en: http://www.quepasa.cl/revista/2002/07/26/t-26.07.QP.CYT.QUIJOTADA.html (consultado 15.02.2005).

Marx, Agnes \& Ernesto Escobar Ulloa (2004). "Interview with Ilan Stavans". Barcelona Review 40. On line en: http://www.barcelonareview.com/40/e_is_int.htm (consultado 12.02.2005).

Pfaff, Carol W. (1979). "Constraints on Language Mixing: Intrasentential CodeSwitching in Spanish/English". Language 55, 291-318.

Poplack, Shana (1980). "Sometimes I'll Start a Sentence in English y termino en español: Toward a Typology of Code-switching". Linguistics 18(7-8), 581-618.

Real Academia Española (2001). Diccionario de la lengua española. 2 vols. Madrid: Espasa-Calpe. 
Roas, David (2005). "Quixotism”. Quimera 252, 9-10.

Rudin, Ernst (1996). Tender Accents of Sound. Spanish in the Chicano Novel in English. Tempe, Arizona: Bilingual Press/Editorial bilingüe.

Ruiz Portella, Javier (2002). "La lengua y la democracia o el Quijote en spanglish". On line en: http://manifiesto.org/spanglish.htm (consultado 12.02.2005).

Sommer, Doris \& Alexandra Vega-Merino (1998). "Either and". Gianna Braschi (ed.). Yo-Yo Boing!. Pittsburgh: Latin American Literary Review Press, $11-18$.

Stavans, Ilan (2003a). Spanglish. The Making of a New American Language. New York: Harper Collins.

Stavans, Ilan (2003b). "Autobiographical Essay". Steven G. Kellmann (ed.). Switching Languages. Translingual Writers Reflect on Their Craft. Lincoln, NE: The University of Nebraska Press, 113-127.

Stavans, Ilan (ed.) (2003c). Wáchale! Poetry and Prose about Growing Up Latino in America. Chicago: Cricket Books.

1 Otros términos, menos afortunados, son español mixtureado o bastardo, argot sajón, slanglish, ingleñol o inglañol, casteyanqui, bilingo, papiamento gringo. Además se distinguen variedades del spanglish como caló pachuco, tex-mex o pocho, cubonics, dominicanish, chicano spanish, nuyorrican, etc. Algunos lingüistas diferencian entre spanglish, que designa al "español anglicado de la frontera méxico-americana" y, más en general, de los inmigrantes hispanohablantes en Estados Unidos, y el espanglés, que "incluye los anglicismos presentes en la terminología especializada del español, como por ejemplo en el léxico relacionado con la economía, la computación, etc." (Fairclough 2003: 187).

2 Franglais era el nombre polémico dado por Étiemble (1964) al francés lleno de anglicismos usado cada vez más en su país. En Canadá, la mezcla de francés e inglés se llama joual (una pronunciación defectiva de la palabra cheval, 'caballo'). El portuñol, a su vez, no sólo es el español defectivo hablado por un lusófono, sino en sentido restringido designa el habla mixta en el área fronteriza entre Argentina y Brasil.

3 El ensayo, tras publicarse en inglés y castellano en varias publicaciones periódicas y páginas web, fue incluido, bajo el título "Mexican Immigration and Hispanization", en Huntington (2004: 221-256).

4 "chopear (tcho-PEHAR), v., 1. to chop" (Stavans 2003a: 97).

5 "employar (em-plo-YAR), v., to employ, to make use" (Stavans 2003a: 113).

6 "sokete (so-KE-te), n., m., sock" (Stavans 2003a: 222). 